\title{
Upgrade of the ATLAS Muon Spectrometer for Operation at the HL-LHC
}

Oliver Kortner on behalf of the ATLAS Collaboration

Max-Planck-Institut für Physik, Föhringer Ring 6, 80805 München, Germany

\begin{abstract}
The High-Luminosity Large Hadron Collider will increase the sensitivity of the ATLAS experiment to rare physics processes. In order to cope with a 10 times higher instantaneous luminosity compared to the LHC, the trigger system of ATLAS needs to be upgraded. The ATLAS experiment plans to increase the maximum rate capability of the $1^{\text {st }}$ trigger level to $1 \mathrm{MHz}$ at $6 \mu$ s latency. This requires new on- and off-chamber electronics for its muon spectrometer. The replacement of the precision chamber read-out electronics will make it possible to include their data in the $1^{\text {st }}$ level trigger decision and thus to increase the selectivity of the $1^{\text {st }}$ level muon trigger. The acceptance of the present RPC trigger system in the barrel region will be increased from $75 \%$ to $95 \%$ by the installation of additional thin-gap RPC with a substantially increased high-rate capability compared to the current RPCs.
\end{abstract}

Keywords: elsarticle.cls, LATEX, Elsevier, template

2010 MSC: 00-01, 99-00

\section{The roadmap to High-Luminosity LHC}

The Large Hadron Collider (LHC) was designed to deliver proton-proton collisions at a centre-of-mass energy of $\sqrt{s}=14 \mathrm{TeV}$ and a luminosity of $1 \cdot 10^{34} \mathrm{~cm}^{-2} \mathrm{~s}^{-1}$. In order to fully exploits the physics potential of the LHC a succesive upgrade to $57 \cdot 10^{34} \mathrm{~cm}^{-2} \mathrm{~s}^{-1}$ is planned: In 2019 and 2020, the so-called "long shut-down 2", the injectors will be upgraded to allow the LHC to deliver its ultimate luminosity of $2 \cdot 10^{34} \mathrm{~cm}^{-2} \mathrm{~s}^{-1}$; the IP region will be upgraded by the installation of new elements including new focusing magnets and crab cavitiesin the years 2024 to 2026 during the so-called "long shut-down 3". The increase of the luminosity from the LHC to the High-Luminosity (HL) LHC by almost an order of magnitude will lead to an increase of particle fluxes by the same amount such that a major upgrade of the LHC experiments for the operation at HL-LHC is required. The topic of this note is the upgrade of the ATLAS muon spectrometer. 


\section{The ATLAS muon spectrometer at the LHC}

15 operated in a magnetic field created by an air-core toroid system to trigger on muons with high transverse momenta $p_{\mathrm{T}}$ up to a pseudorapidity $|\eta|=2.4$ and to measure $p_{\mathrm{T}}$ with $4 \%$ resolution in a wide momentum range and $10 \%$ at $p_{\mathrm{T}}=1 \mathrm{TeV}$ up to $|\eta|=2.7$ [1]. Resistive Plate Chambers (RPC) in the barrel region and Thin nanoseconds for $p p$ bunch crossing identification, but moderate spatial resolution are used for the first level trigger. The high muon momentum resolution is achived by a precise measurement of muon trajectories with Cathode Strip Chambers (CSC) in the inner end-cap disk at large rapidities and Monitored Drift Tube (MDT) chambers in the rest of the spectrometer. These chambers have spatial resolutions better than $40 \mu \mathrm{m}$.

ATLAS uses a 3-level trigger system. A high- $p_{\mathrm{T}}$ muon trigger built out of a conincidence of three RPCs in the barrel toroid magnet and three TGCs behind the end-cap toroid magnet is part of the first trigger level. The muon momentum is estimated from the sizes of the deviations of the trigger chamber hits from a straight line from the $p p$ interaction point.

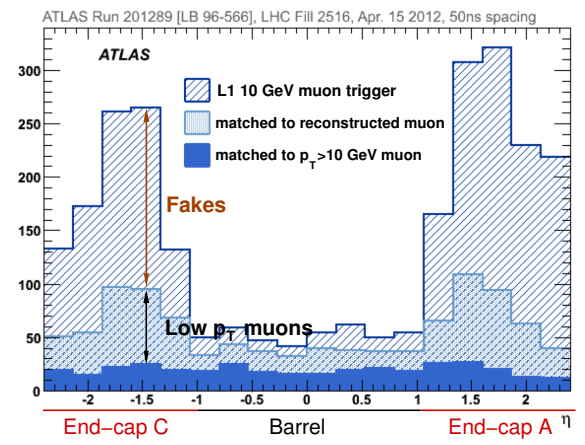

Figure 1: Contriubtions to $1^{\text {st }}$ level muon triggers for a $10 \mathrm{GeV}$ transverse momentum threshold as a function of $\eta$ in LHC Run 1. [2]

Figure 1 shows the distribution of $1^{\text {st }}$ level muon triggers for a $10 \mathrm{GeV}$ transverse momentum threshold as a function of $\eta$ in LHC Run 1. The trigger rate is dominated by triggers in the end-cap regions. A large fraction of these triggers are caused by charged particles, mainly protons, emerging from the radiation shielding and the materials of the end-cap toroid into the spectrometer where they leave traces in the TGCs behind the toroid magnetic which look like high- $p_{\mathrm{T}}$ muons from the interaction point. These fake triggers can be rejected by taking trigger chambers in 
front of the end-cap toroid magnet into the $1^{\text {st }}$ level concidence. After the removal of fake triggers the main source of muon triggers are muons with $p_{\mathrm{T}}<10 \mathrm{GeV}$ which are selected due to the poor momentum resolution at level 1 caused by the moderate spatial resolution of the trigger chambers.

\section{The ATLAS muon spectrometer at the HL-LHC}

The plan for the upgrade of the ATLAS muon spectrometer 3 is illustrated in Figure 2

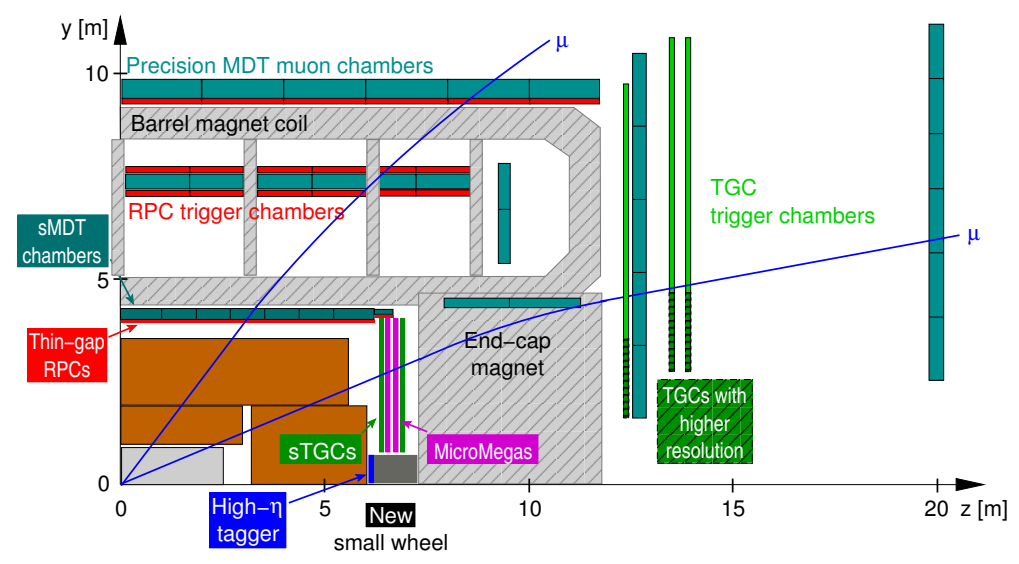

Figure 2: Schematic drawing of a quadrant of the ATLAS muon spectrometer illustrating the planned upgrade for HL-LHC.

The ATLAS muon spectrometer is operated in a large background of neutrons and $\gamma$ rays. In order to cope with background counting rates of up to $15 \mathrm{kHz} \mathrm{cm}^{-2}$ the so-called "small wheel" will be replaced by a new small wheel (NSW)with chambers with increased high-rate capability in the long shutdown 2 [2]. The other muon spectrometer upgrades will be carried out in long shutdown 3. In the NSW small strip TGCs (sTGCs) will be used for triggering while MicroMegas will be used for precision tracking and a refined muon momentum measurement at trigger level. The big wheel's TGCs closest to the beam pipe will be replaced with TGCs with higher spatial resolution to increase the selectivity of the $1^{\text {st }}$ level muon trigger.

55 New thin-gap RPCs will be added to the inner barrel layer to close acceptance gaps of the barrel muon trigger. To free space for the RPCs MDT chambers will need to be replaced by so-called "sMDT chambers" which are drift-tube chambers with $15 \mathrm{~mm}$ diameter tubes instead of $30 \mathrm{~mm}$ diameter tubes. In order to identify muons within the whole acceptance of the upgraded inner detector of $|\eta| \leq 4.0$ a muon tagger will be added close to the beam pipe between the forward calorimeter and the 
shielding disk of the NSW. Finally the new trigger architechture will require new on- and off-chamber electronics.

\section{Upgrade of the $1^{\text {st }}$ level muon trigger system}

The ATLAS experiment will adopt a multilevel trigger system also for the data acquisition at the HL-LHC. Yet, to allow for more sophisticatic algorithms the latency of the first trigger level will be increased by about $3 \mu \mathrm{s}$ to $6 \mu \mathrm{s}$. The enlarged latency makes it possible to include the MDT chamber data in the $1^{\text {st }}$ level muon trigger decision for a better $p_{\mathrm{T}}$ resolution and selectivity.

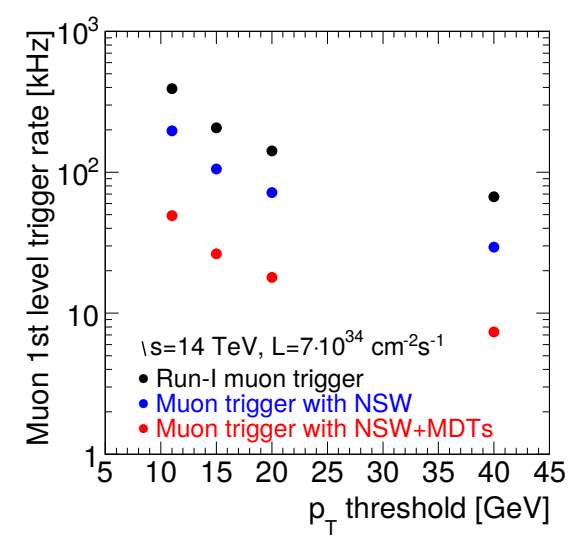

Figure 3: Single muon trigger rates at the HL-LHC luminosity of $7 \cdot 10^{34} \mathrm{~cm}^{-2} \mathrm{~s}^{-1}$ for three different scenarios: the Run-1 muon trigger, the Run-1 muon trigger with the NSW, the final trigger with the NSW and the MDT chamber data [2, 3].

This requires new MDT front-end electronics for streaming the MDT hit data

off the chambers to new muon trigger processors where they will be used for a refined muon momentum measurement. It was demonstrated with data recorded in CERN's Gamma Irradiation Facility that muon tracks in MDT chambers can be reconstructed with an algorithm seeded by the RPC and TGC triggers within $3 \mu \mathrm{s}$ on a ARM Cortex A9 CPU running at $1 \mathrm{GHz}$.

Figure 3 shows the expected single muon trigger rate as a function of the $p_{\mathrm{T}}$ threshold for three scenarios: the Run-1 muon trigger, the Run-1 trigger with the NSW in conincidence, and the HL-LHC muon trigger which also uses the MDT data. The inclusion of the NSW reduces the trigger rate by a factor 2, the MDT trigger by another factor of 3 such that a very small single-muon trigger rate of about $18 \mathrm{kHz}$ for a $p_{\mathrm{T}}$ threshold of $20 \mathrm{GeV}$ is expected at the HL-LHC. 


\section{References}

[1] ATLAS-Collaboration, The atlas experiment at the cern large hadron collider, JINST 3 (2008) S08003.

[2] ATLAS-Collaboration, New Small Wheel Technical Design Report, Tech. Rep. CERN-LHCC-2013-006. ATLAS-TDR-020, CERN, Geneva (2013).

[3] ATLAS-Collaboration, ATLAS Phase-II Upgrade Scoping Document, Tech. Rep. CERN-LHCC-2015-020. LHCC-G-166, CERN, Geneva (2015). 